\title{
Cellular Desmoplastic or Anaplastic Supratentorial Ependymoma with Neuronal Differentiation in Probable Associated to RELA Fusion? Case Report
}

\author{
Martha Lilia Tena-Suck ${ }^{1 *}$, Armando Ruiz-Treviño², Laura Chávez-Macías ${ }^{3}$, Carlos Peñafiel-Salgado ${ }^{1}$ \\ and Carlos Sánchez-Garibay ${ }^{1}$
}

${ }^{1}$ Departamento de Neuropatología, Instituto Nacional de Neurología y Neurocirugía, México

${ }^{2}$ Servicio de Neurocirugía, Instituto Nacional de Neurología y Neurocirugía, México

${ }^{3}$ Departamento de Patología, Hospital General de México, México

*Corresponding author: Martha Lilia Tena-Suck, Departamento de Neuropatología, Instituto Nacional de Neurología y Neurocirugía, Cuidad de México "Manuel Velasco Suarez", Av. Insurgentes Sur 3877 Col. La Joya, Delegación Tlalpan, 14269, México City, México, Tel: (0155)-56063822, Fax: (525)-54240808

\begin{abstract}
Cellular Ependymoma is a term losing in the new WHO 2016 criteria, actually are classified as classical or anaplastic or associated to RELA fusion. We presented a rare case report of classical cellular ependymoma in supratentorial location, which presented a neuronal differentiation and desmoplasia in a young man of 29-yr-old, Immunohistochemistry tumor cells expressed Neu-N, Synaptophysin, SNE, $\alpha$-synuclein, beta tubulin, ubiquitin, neurofilament, D2DR, and GFAP and vimentin. This rare tumor is a very controversial. It was classified as intermediated grade and recently is named as anaplastic or associated to RELA fusion with neuronal differentiation and with dense and diffuse desmoplastic stroma. Genetic expression of RELA gene was required to make a better diagnostic according with the new classification of Central Nervous System classification according with the WHO.
\end{abstract}

\section{Keywords}

Ependymoma, Cellular ependymoma, WHO classification, Neuronal differentiation, Desplomasia

\section{Abbreviations}

EP: Ependymoma; WHO: World Health Organization; AEP: Anaplastic Ependymoma; L1CAM: Neuronal Cell Adhesion Molecules; GFAP: Glial Fibrillary Acidic Protein; EMA: Epithelial Membrane Antigen; MRI: Magnetic Resonance Imaging; NSE: Neuron Specific Enolase; D2DR: Polyclonal
Dopamine D2 Receptor; SMA: Specific Muscle Actin; HCG: Human-Chorionic-Gonadotropin; ACE: Antigen Carcioembrionary; NTRK1: Neurotrophins Receptors TrkA; NGFRAP1: Nerve Growth Factor Receptor-Associated Protein 1; Neu-N: Pan-Neuronal Markers; GJA1: Junctional Protein Connexin 43

\section{Introduction}

Ependymoma (EP) is a tumor of children and young adults originating from the wall of the ventricles or from the spinal canal and composed of neoplastic ependymal cells, and account for 2-9\% of all neuroepithelial tumors [1]. The subtype called EP cellular is a rare variant common in extraventricular location, shows conspicuous cellularity with pseudo-rosettes but no true rosettes may present [1]. This variant is classified as WHO II [1]. Tumor Location has been identified as an important prognosis factor. The supratentorial site is associated with a better prognosis than posterior fossa neoplasm. EPs are considered conferring to their locations and genetic abnormalities. The most important predictive prognostic factors for ependymoma is genomic aberration [1]. However recently with the new WHO classification, neither histological type or tumor grade or tumor location are associated with better prognosis, and these

Citation: Tena-Suck ML, Ruiz-Treviño A, Chávez-Macías L, Peñafiel-Salgado C, Sánchez-Garibay C (2019) Cellular Desmoplastic or Anaplastic Supratentorial Ependymoma with Neuronal Differentiation in Probable Associated to RELA Fusion? Case Report. Int J Pathol Clin Res 5:087. doi.org/10.23937/24695807/1510087

Accepted: April 18, 2019: Published: April 20, 2019

Copyright: (C) 2019 Tena-Suck ML, et al. This is an open-access article distributed under the terms of the Creative Commons Attribution License, which permits unrestricted use, distribution, and reproduction in any medium, provided the original author and source are credited. 
two parameters are important prognostic factors for patient outcome [1]. As a result, the difficulty in assigning clinical significance to ependymoma histological grades is discussed in the grading sections of both the EP and Anaplastic Ependymoma (AEP) and incorporation of a genetically defined ependymoma variant RELA fusion entity [1]. Though, several conflicting reports regarding patients' outcomes have been reported $[2,3]$.

EP has been divided into three groups conferring to their location: Supratentorial (ST), posterior fossa $(\mathrm{PF})$, and supratentorium and spinal cord (SC). The STEpendymoma (ST-EP) is further categorized as RELA-C11 or f95 fusion and YAP1-MAMDL1 or YAP1-FAM118B fusion [4]. For ST-EP, RELA fusion-positive ependymoma has been showed a more intense or aggressive comportment than YAP1 fusion-positive ependymoma [4]. The specificity of L1CAM expression, a potential immunohistochemical alternate for this variant [4]. RELA fusion can be detected via reverse transcriptasepolymerase chain reaction or immunohistochemical marker L1CAM [4-6]. Those tumors usually expressed GFAP and EMA. Cellular ependymoma variant has been removed from the classification since it was considered to overlap lengthily with standard ependymoma $[1,4]$. The aim of this clinical presentation case report is in relation a case of supratentorial ependymoma with focal anaplasia and with neural differentiation and desmoplastic features. Called as cellular ependymoma and with the recently WHO classification called as anaplastic ependymoma with neuronal differentiation associated to variant RELA fusion entity $[1,5,6]$.

\section{Clinical Presentation}

29-years-old man, no history of importance, starts his suffering for a month with a headache, which it increased with the effort and the exercise. Added right intraocular pain, besides nausea, vertigo, vomiting, diplopia, blurred vision, photophobia and right facial synesthesia.

Physical examination revealed no abnormality, with a normal neurological examination. Magnetic resonance imaging (MRI) scan showed a hypodense area in the right temporal region, with marked enhancement in the medial parts and severe surrounding brain edema (Figure 1).

The specimen, which was received in the pathology department, showed multiple fragments of grayish and firm tissue, altogether measuring about $6 \mathrm{~cm} \times 5$ $\mathrm{cm} \times 3 \mathrm{~cm}$. Histological features revealed a markedly desmoplastic tumor, showing deposition of dense collagen fibers (Figure 2a and Figure 2b). Scattered small, round and blue cells were also observed (Figure $2 c$ and Figure $2 d$ ), the monotonous population was seen with a malignant phenotype. Some mitosis and necrosis were present. Endothelial proliferation and necrosis were also seen. The neoplastic cell population was heterogeneous, composed of spindle-shaped cells with a fascicular arrangement in the abundant fibrous and collagenous (Figure 2e) and reticulin-rich stroma (Figure 2f). On immunohistochemistry indicating neuronal differentiation, cells were positive immunoreaction to GFAP (Figure 3a), Neu-N (Figure 3b), synaptophysin, and NSE and Neurofilament (Figure 3c), vimentin (Figure 3d), EMA in
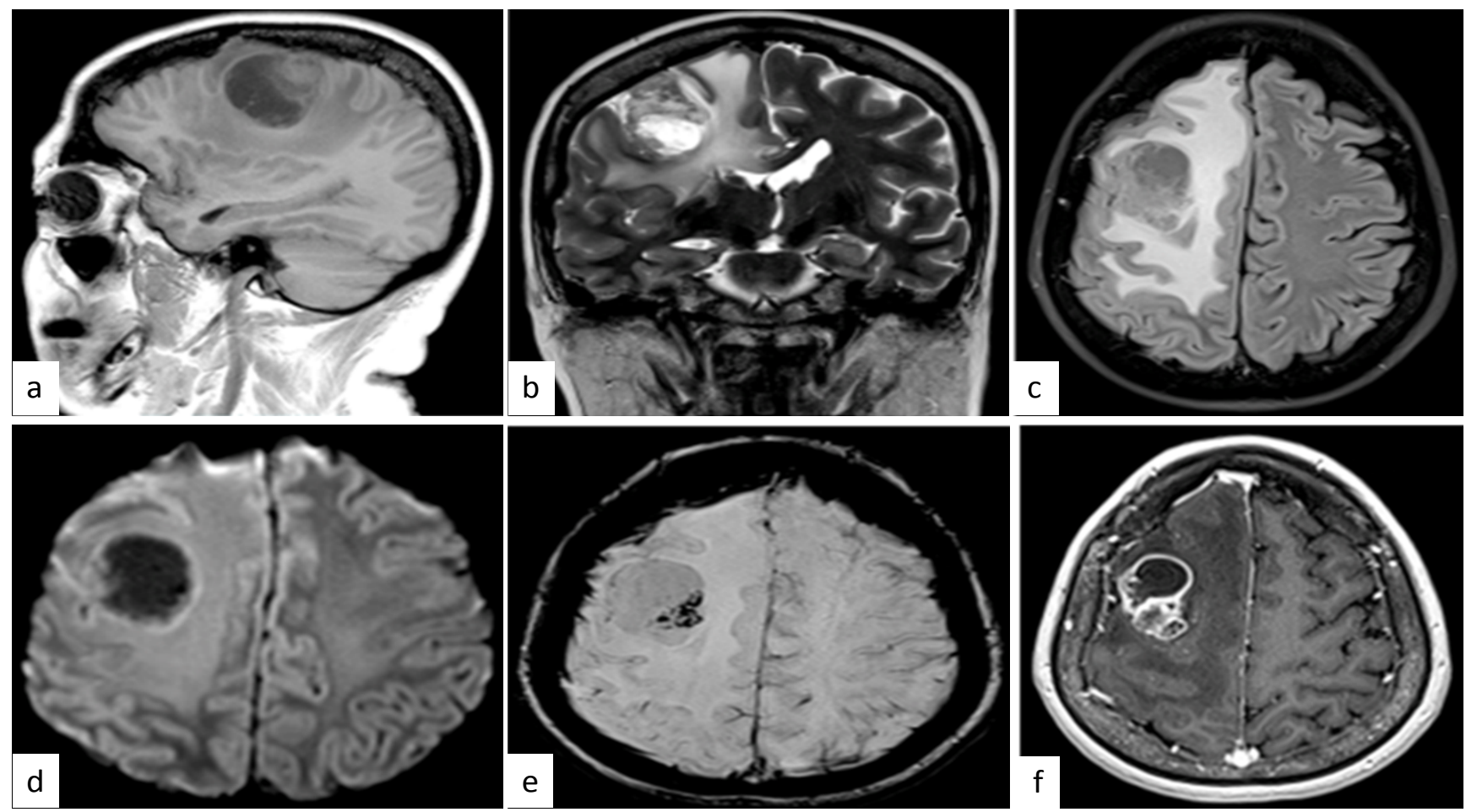

Figure 1: Radiologically imagines. (a), (b) and (c). MRI showed a brain tumor with hypodense area in the right temporal region (d), (e) and (f) with marked enhancement in the medial parts and severe surrounding brain edema. 

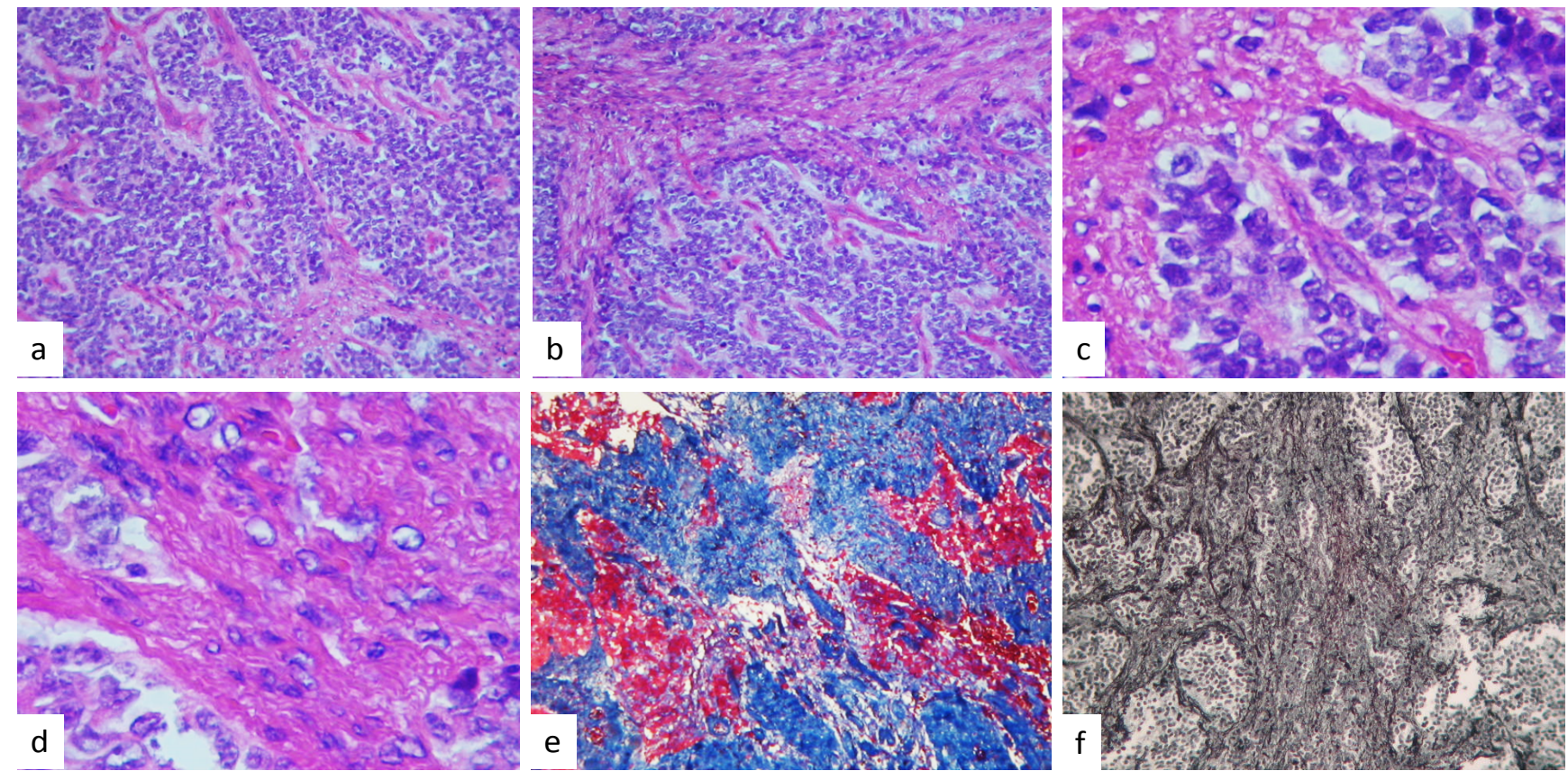

Figure 2: Histologically tumor formed a markedly desmoplastic tumor, showing deposition of dense collagen fibers in (a) and (b). The neoplastic cell population was heterogeneous, composed of spindle-shaped cells and small. Round and blue cells with a fascicular arrangement in the abundant collagenous stroma were observed in (c). Showed the cellular monotonous population in the desmoplastic areas seen to be the same cellular phenotype in (d). With Masson stain observed in blue the most part of the tumor in (e) and in (f) observed reticulin-rich stroma (original magnification $\times 400$ ).
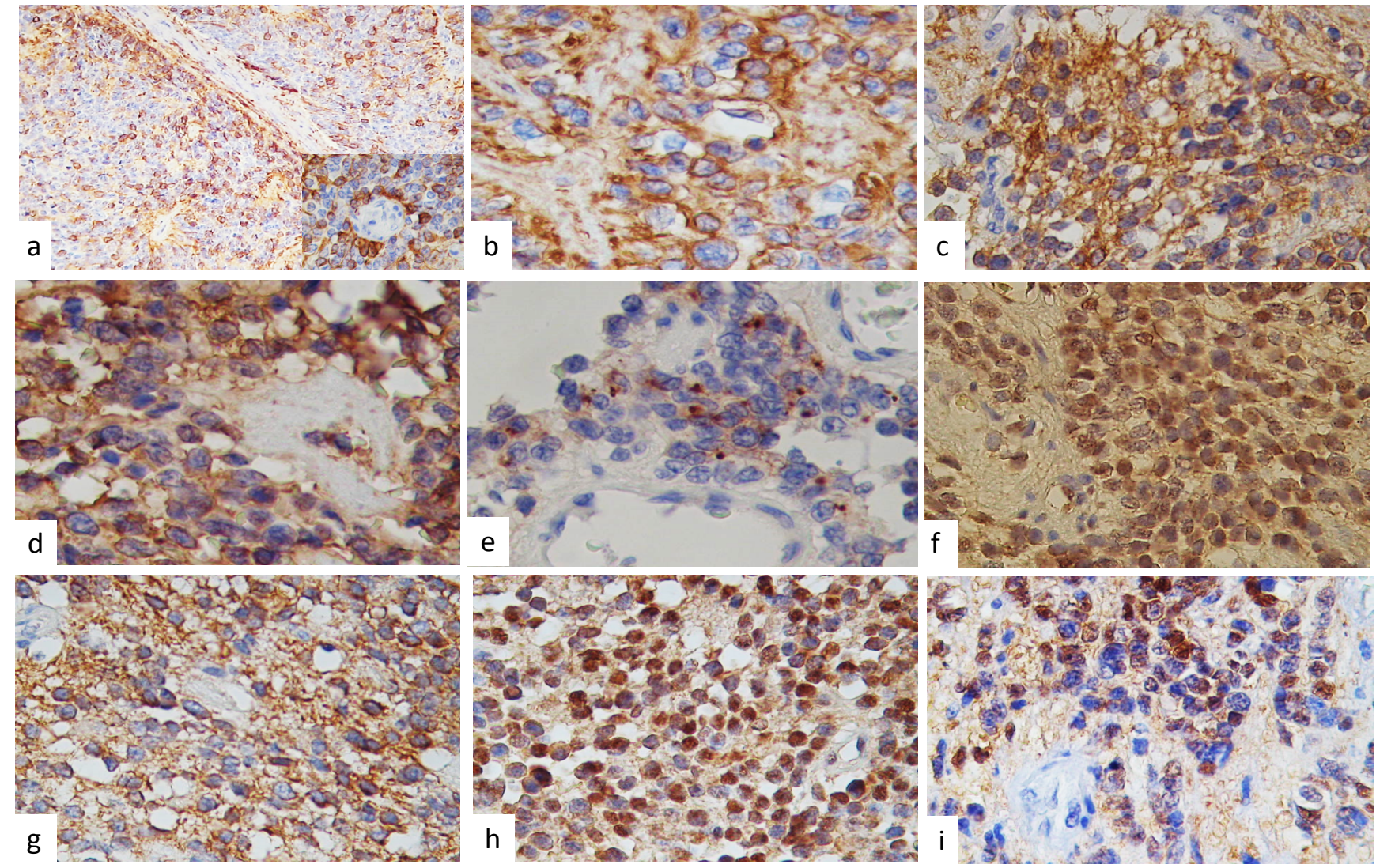

Figure 3: On immunohistochemistry indicating neuronal differentiation, cells were positive immunoreaction to GFAP (a), Neu-N (b), NSE in (c), vimentin (d), EMA in punctate pattern in (e), Tao proteins were nuclear positive reaction (f), ubiquitin showed a fibrillary pattern in $(\mathrm{g})$, alpha-synuclein with nuclear expression (h) and D2DR Selectively distribution in (i) (IHQ stain $\times 400$ ).

punctate pattern (Figure 3e), Tao proteins were nuclear positive reaction (Figure $3 f$ ), ubiquitin with fibrillary pat- tern (Figure 3g), alpha-synuclein with nuclear expression (Figure 3h) and D2DR Selectively distribution (Fig- 
ure 3i). MIB-1 (Ki-67) labeling index revealed a proliferation rate of $40 \%$. Keratin $7 / 8 / 20$ and AE6/AE7. RELA by immunohistochemistry was focal positive immunoreaction. SMA, myogenin, desmin, EMA, HCG, and $\alpha$-fetoprotein, $A C E, C D 99, C D 20$ staining remained negative. A preliminary diagnosis of the desmoplastic tumor was made. Finally, the cellular ependymoma desmoplastic with neuronal differentiation was diagnosed as well as, anaplastic desmoplastic ependymoma with neuronal differentiation associated to variant RELA fusion entity according to with 2016 WHO criteria [1].

\section{Discussion}

Ependymomas (EPs) are glial neoplasms arising in any location throughout the CNS and evidently, are derived from radial glia cells. Current data propose that these tumors may have different biological and clinical behaviors according to their location, characterized histologically by medium sized, poorly differentiated cells with round or oval nuclei frequently in mitosis and by rosettes or pseudo rosettes formations. Histological restrictions, such as mitotic rate, microvascular proliferation, and nuclear pleomorphism. Nevertheless, it is trying to apply and the clinical utility is uncertain. Positivity immunoreaction for vimentin and S-100 protein, reactions for cytokeratin and neurofilament are usually negative and tubular material positive for GFAP focal or scanty and [4]. The grouping of mixed myoneural tumors of the CNS has been rapidly losing its definition as encompassing tumors composed of histologically distinct neuron variants and glia [4]. We presented a rare case of a classical histological EP with a two different histological patterns; small, round and blue cells with focal anaplasia and mitosis features and strong desmoplasia, also immunohistochemistry expression showed neural differentiation [3]. The problem that we have is how to call or diagnosed this tumor; as cellular desmoplastic ependymoma or supratentorial anaplastic ependymoma with neuronal differentiation according to with the new WHO 2016 classifications criteria [1,6]. This rare neoplasia which they were called at any time as emerging tumor entities and variants of CNS neoplasms has been recently divided into three groups according to their location and their genomic aberration: supratentorial, posterior fossa and spinal cord. ST Ependymo$\mathrm{ma}$ is additional categorized as RELA-C11 or $\mathrm{f95}$ fusion and YAP1-MAMDL1 or YAP1-FAM118B fusion [5]. In case of Posterior Fossa ependymomas, LAMA2-expressing ependymomas has been seen worse outcome than NELL2-expressing ependymomas $[5,6]$. Combinations between RELA, which encodes an NF-KB component, and the poorly-characterized gene $\mathrm{C} 11$ orf95 produced by a local chromosome devastating event (chromothripsis) on chromosome 11 were seen in $>70 \%$ of supratentorial ependimomas. That corresponding an aberrantly expressed in neural stem cells [6].

We presented a rare case of ST-EP with a conclu- sive and extraordinary histology, with neural differentiation and no genetic analysis was confirmed as RELA fusion tumors. L1CAM expression was no performance in this case. We don't have the arguments to call it as RELA- fusion entity. We presented a rare case report that clinical and histological criteria could complete all of RELA fusion EP criteria [1]. However, the histological features presents of this case are sufficient and interesting to comment. This is a supratentorial tumor location, histologically with small, round and blue cells with and robustly desmoplastic stroma [1]. Immunohistochemical stain, it was positive immunoreaction to $\mathrm{Neu-N}$, synaptophysin, NSE, nestin, neurofilament, vimentin, and GFAP. This is a rare histological variant of brain tumor with neuronal differentiation $[4,7]$. Recent studies revealed that some supratentorial ependymomas express neuronal antigens and that high expression of neurofilament protein light polypeptide (NEFL) neurotrophins receptors TrkA (NTRK1) and p75 (NGFR), pan-neuronal markers Neu-N (RBFOX3) and synaptophysin, radial glial marker SOX9, adhesion molecules CD56 (NCAM) and CD44, junctional protein connexin 43 (GJA1), and GFAP, which conditional on tumor topography supporting the interpretation of divergent cells of origin or stem cells [8]. Neutral glycolipid and gangliosides compositions [9], as well as, Alpha-synuclein [10], can reveal neuronal phenotypes and have been reported a relationship between the degrees of neuronal differentiation in tumors of mixed tumors of the CNS [1]. The authors presented a rare case with Alpha-synuclein, Tao protein, tubulin, ubiquitin, neurofilament and D2DR immunoexpression.

Histologically, the prominent feature of all tumors is a striking desmoplastic stroma and in combination with variable neuroepithelial and fibroblastic elements. Part of the spindle cells were immunoreactive for GFAP. Highly cellular areas of undifferentiated cells with frequent mitotic figures and necrosis, the so-called anaplastic areas, can also be seen [7].

The differential diagnosis that we have to consider including; desmoplastic infantile Ganglioglioma, desmoplastic small cells tumor, gliosarcoma, primitive neuroectodermal tumor, rhabdomyosarcoma, and Wilms' tumor, as well as, desmoplastic neuroepithelial tumor and also fourth ventricle neurocytomas with lipomatous and ependymal differentiation $[4,7]$.

Supratentorial superficially located cystic neuroepithelial tumors of infancy characterized by prominent desmoplasia with neoplastic glial component (desmoplastic infantile astrocytoma) or neoplastic glioneuronal component (desmoplastic infantile Ganglioglioma), corresponds to WHO grade I, they usually expressed neuroepithelial component and GFAP: Positive, vimentin with variable expression, GFAP positive and neuronal markers (e.g. synaptophysin, NSE, Neu-N) positive if ganglion cells present $[4,7]$. The first element can be revealed to be GFAP positive, but the latter element is 
of neuroepithelial origin and reactive with markers such as synaptophysin, NSE, Neu-N [7]. Neuronal differentiation occurs in ependymomas but is less frequently decisive (histologic, ultrastructural) than simply is restricted immunohistochemical finding $[4,10]$.

The main histologic differential diagnoses are reticulin-rich desmoplastic tumors such as pleomorphic xanthoastrocytoma, which can be differentiated by age of the patient, prominent lipidization of the cells and absence of the neural component. Another tumor in this category is gliofibroma and gliosarcoma which lacks the neural component [1].

Desmoplastic small round cell tumors are a type of soft tissue sarcoma (DSRCT) shares characteristics with other small and round blue cell cancers including Ewing's sarcoma, acute leukemia, small cell mesothelioma, neuroblastoma, primitive neuroectodermal tumor, rhabdomyosarcoma, and Wilms' tumor. On immunohistochemistry, these cells have coexpression with the epithelial marker cytokeratin, the mesenchymal markers desmin and vimentin, and the neuronal marker as synaptophysin, NSE, and Neu-N [10].

\section{Conclusion}

EPs are common pediatric and adult CNS malignancies with a wide biologic spectrum that is often hard to predict using classic prognostic variables. The molecular pathogenesis is also poorly understood. The category of mixed glio neuronal tumors of the CNS is rapidly losing its definition as encompassing tumors composed of histologically distinct neuron variants and the clinical significance of these observations is unknown but deserves further exploration or it is hypothesized to arise from a progenitor cell with multi-phenotypic differentiation.

\section{Author Contributions}

Conceptualization: MLTS, LCHM

Data curation: MLTS, CP, LCHSM

Formal analysis: CP, LCHM, ART

Funding acquisition: MLTS, LCHM, CSL, CSG, ART

Investigation: MLTS, ART, CSG

Methodology: MLTS, ART, CSG, and CPS.

Project administration: MLTS

Resources: MLTS, ART, CSG, and CPS.

Software: CPS

Supervision: MLTS

Validation: MLTS, CSG and ART

Visualization: MLTS, ART, LCHM, CSG, and CPS.

Writing - original draft: MLTS, CPS, LCHM

Writing - review \& editing: MLTS, CPS.

\section{Conflict of Interest Statement}

The author declares that the research was conducted in the absence of any commercial or financial relationships that could be construed as a potential conflict of interest.

\section{Funding}

We had no funding for this study.

\section{Disclosure}

We have no conflict of interest to disclose.

\section{Competing Interests}

The authors have declared that no competing interest exists.

\section{Acknowledgements}

We thank Dr. Fausto Rodriguez for his valuable contribution in the diagnosis of this case, and also we are grateful to our research study assistants Neomi Gelista and Brenda Peralta in the Neuropathology Department, for their technical expertise and assistance.

\section{Competing Interests}

The authors have declared that no competing interest exists.

\section{References}

1. Louis NL, Perry A, Reifenberger G, Von Deimling A, Figarella-Branger D, et al. (2016) The 2016 world health organization classification of tumors of the central nervous system: A summary. Acta Neuropathol 131: 803-820.

2. Cenacchi G, Giangaspero F (2004) Emerging tumor entities and variants of CNS neoplasms. J Neuropathol Exp Neurol 63: $185-192$

3. Per H, Kontaş O, Kumandaş S, Kurtsoy A (2009) A report of a desmoplastic non-infantile ganglioglioma in a 6-year-old boy with review of the literature. Neurosurg Rev 32: 369374.

4. Rodriguez FJ, Scheithauer BW, Robbins PD, Burger PC, Hessler RB, et al. (2007) Ependymomas with neuronal differentiation: a morphologic and immunohistochemical spectrum. Acta Neuropathol 113: 313-324.

5. Gajjar A, Bowers DC, Karajannis MA, Leary S, Witt $H$, et al. (2015) Pediatric brain tumors: innovative genomic information is transforming the diagnostic and clinical landscape. J Clin Oncol 33: 2986-2998.

6. Pajtler KW, Witt H, Sill M, Hovestadt V, Kratochwil F, et al. (2015) Molecular classification of ependymal tumors across all CNS compartments, histopathological grades, and age groups. Cancer Cell 27: 728-743.

7. Andreiuolo F, Puget S, Peyre M, Dantas-Barbosa C, Boddaert N, et al. (2010) Neuronal differentiation distinguishes supratentorial and infratentorial childhood ependymomas. Neuro Oncol 12: 1126-1134.

8. Hagel C, Treszl A, Fehlert J, Harder J, Von Haxthausen F, et al. (2013) Supra- and infratentorial pediatric ependymomas differ significantly in NeuN, p75 and GFAP expression. J Neurooncol 112: 191-197. 
9. Yates AJ, Franklin TK, McKinney P, Collins R, Comas T, et al. (1999) Gangliosides and neutral glycolipids in ependymal, neuronal and primitive neuroectodermal tumors. J Mol Neurosci 12: 111-121.
10. Raghavan R, White CL III, Rogers B, Coimbra C, Rushing EJ (2000) Alpha-synuclein expression in central nervous system tumors showing neuronal or mixed neuronal/glial differentiation. J Neuropathol Exp Neurol 59: 490-496. 